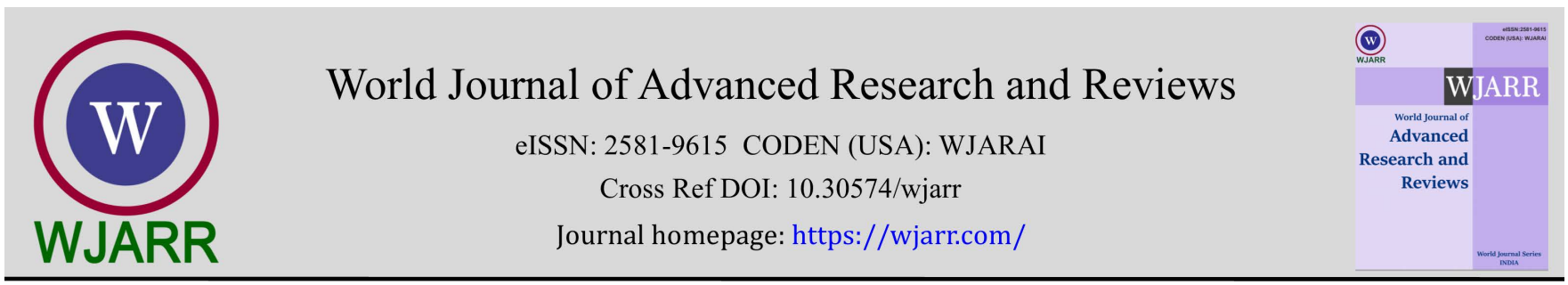

(RESEARCH ARTICLE)

\title{
Cognitive ageing: 20 years process
}

\author{
Gülbün Asuman Yüksel ${ }^{1}$ and Gizem Gürsoy 2,* \\ ${ }^{1}$ Neurology Department, Haydarpaşa Numune Training and Research Hospital, İstanbul, Turkey. \\ ${ }^{2}$ Neurology Department, Ümraniye Training and Research Hospital, İstanbul, Turkey.
}

World Journal of Advanced Research and Reviews, 2021, 12(03), 304-312

Publication history: Received on 08 November 2021; revised on 14 December 2021; accepted on 16 December 2021

Article DOI: https://doi.org/10.30574/wjarr.2021.12.3.0692

\begin{abstract}
Background: People with normal functionality have normal cognitive changes associated with the ageing process while many people age without cognitive decline. The most exact effects of age are cognitive impairments in learning, memory, and problem solving. These age-related effects slightly increase or do not change for many years, and do not affect the daily life activities of the person.
\end{abstract}

Methods: To investigate age-related cognitive effects, detailed cognitive evaluations were compared with 20 years intervals in 7 (seven) elderly individuals at Haydarpaşa Numune Training and Research Hospital in 2019. These individuals are continuing daily life activities and sociocultural relations independently. Mini-mental state examination, verbal memory processes test and visual memory test-Wechsler memory scale for memory processes, digit span test for attention function, verbal fluency, similarities, stroop, and trail-making test for the evaluation of frontal lobe functions, Benton's line direction determination test for the visuospatial organization have been applied to individuals.

Results: In comparison with the cognitive test results applied twenty years ago; immediate memory impairment is evident, abstraction and attention function are relatively less affected. The tests showing the frontal lobe function, the verbal fluency which also reflects the vocabulary information is less affected, while the cognitive impairment is more in consecutive-complex processes.

Conclusion: Cognitive functions based on attention, vocabulary and knowledge are substantially preserved with mild improvement in normal ageing. The most important improvement is on executive functions due to the decrease in motor and cognitive processing speed in cases where complex information needs to be processed.

Keywords: Ageing; Cognitive ageing; Normal cognition; Cognitive function

\section{Introduction}

The phenomenon of cognitive ageing has been noticed almost as long as the phenomenon of physical ageing, but it is still not well understood. This is unfortunate because cognitive functioning can affect one's quality of life, and even the ability to live independently. Furthermore, cognitive functioning in early adulthood may be related to the development of pathologies such as Alzheimer's disease in later adulthood. Elderly people, even middle-aged people, complain of difficulty remembering names, faces, birthdays, meetings and other events in everyday life. Normal cognitive ageing is defined as the ageing changes that occur in individuals without any other diseases of the nervous system [1]. Cognitive decline, although a relatively common occurrence, cannot be considered an inevitable part of ageing [2-5].

\footnotetext{
* Corresponding author: Gizem Gürsoy

Neurology Department, Ümraniye Training and Research Hospital, İstanbul, Turkey.

Copyright $(2021$ Author(s) retain the copyright of this article. This article is published under the terms of the Creative Commons Attribution Liscense 4.0.
} 
While the cognitive decline that occurs with ageing does not reach a level that will affect daily life and social relations in some people, it may cause neurodegenerative diseases (dementias) with severe cognitive impairment in others. While these cognitive changes may vary from person to person, different cognitive abilities may be affected at different levels in the same person [6]. It is increasingly important to answer whether the decline in cognitive abilities seen with ageing is a normal consequence of old age or an early sign of a neurodegenerative disease such as Alzheimer's disease $[4,6-8]$.

Cognitive ageing is not dementia and does not result in the loss of neurons but rather changes in neurotransmission that affects brain functioning. The fact that neurons are structurally intact, but functionally affected by increasing age means that this pathological process has the potential to be prevented or cured $[5,9]$.

Researchers have proposed various mechanisms to explain patterns of age-related changes; these include reduced processing resources, loss of inhibitory functions, and lack of cognitive control and general slowing [10]. Information processing speed refers to the speed at which cognitive abilities are realized, as well as the speed of motor responses. This ability begins to decline in the third decade of life and continues to decline throughout life. Therefore, the time to learn new information is prolonged and more cognitive effort is required to acquire new information [11]. Most of the cognitive changes reported in healthy older adults are the result of slowed processing speed. This slowdown can adversely affect performance on many neuropsychological tests designed to measure other cognitive domains (ie. verbal fluency). Therefore, this decrease in processing speed can manifest itself in various cognitive areas [3,12].

The normal ageing process is associated with a significant reduction in brain volume, especially in the frontal cortex, with other regions such as temporal, parietal, subcortical, cerebellar. This explains the poor performance in tests showing frontal lobe function. There is considerable regional specificity within the cerebral cortex and the hippocampus in both the degree and cellular basis for loss[12,13]. The prefrontal cortex is more sensitive to the effects of ageing than the hippocampus and is more affected. A loss of neurons, dendrites and synapses has been documented, as well as changes in neurotransmitter systems, in some regions of the cortex and hippocampus but not others [14-18]. The relationship between structural linkage degeneration that occurs with normal ageing and cognitive decline remains unclear[16,19,20].

Age is the largest risk factor for the development of neurodegenerative syndrome, and more than one-third of clinically normal adults can have significant clinicopathological changes at autopsy. The ageing process occurs progressively and cumulatively [4]. Lipofuscin accumulation, gliosis and neuronal degeneration are seen in neurons. Glial cell reactivation, retraction and expansion in dendrites, synapse loss and reorganization occurs with the accumulation of insoluble proteins such as tau, amyloid and alpha-synuclein in the extracellular area. In this process where the brain volume decreases, the neuronal dendrites especially in the 3rd and 4th layers of the neocortex decrease in the ageing brain, and the affected neurons form a new expanded dendritic network. This supports that even older neurons can develop new synapses in response to cell loss [5,6,15,20,21].

Cell loss in the limbic system is particularly associated with memory loss. Studies have shown that the loss of cells in the hippocampus is approximately $27 \%$ between the ages of 45-95. This decrease in brain volume accelerates from 60 years. Whether or not they will develop into dementia in the future, normal people begin to develop neocortical plaques and limbic neurofibrillary tangles from the age of 60. During normal ageing, a correlation has been shown between the limbic (entorhinal and hippocampal) neurofibrillary tangle count and chronological age. Not all groups of neurons are equally affected. With ageing, changes in neurotransmitter systems may occur without cell damage [6,20,21].

Studies in healthy older adults show declines in some cognitive areas, while others improve on the contrary. One of the most common cognitive complaints among older adults is the alteration in memory. The complaint of forgetfulness, also known as subjective memory complaint, is quite common among 25-56\% of the elderly. Age-related memory changes may be related to slowing processing speed, decreased ability to ignore irrelevant information, and reduced use of strategies to improve learning and memory. As a result, older adults experience greater difficulties in their memoryrelated tasks, and they have to act more complex, troublesome and strategic $[3,10]$.

Memory reported by Tulving has five separate components that interact with each other; procedural memory, perceptual representation system, semantic memory, working memory and episodic memory. Ageing affects some types of memory more $[8,10]$. Most commonly, healthy older adults show impairments in attention, working memory, and episodic memory tasks compared to younger adults. Short-term memory is affected by age. In long-term memory, the impairment of spontaneous recall performance is more severe than recognition and clue recall performance. While episodic memory is significantly impaired, semantic memory and procedural memory are partially preserved. However, 
older adults display much better performance than young people and young adults on tests that assess fund of knowledge and general knowledge [1,7,22,23].

General language aptitude remains intact with ageing. Vocabulary stays constant and even improves over time. Different dimensions of attention (such as divided attention-selective attention) are negatively affected by ageing and failure to maintain attention (concentration) is observed $[2,3]$.

Impairment-failure is observed in cognitive flexibility and visual-spatial functions such as three-dimensional structuring and shape duplication [11].

It is advocated that maintaining an active lifestyle and engaging in certain activities throughout a person's life can help prevent age-related cognitive decline [3,24].

\section{Methods}

At 20 years follow-up; 20 healty people had detailed cognitive test. After stroke and malignancy-related deaths; the same tests were administered to seven healthy individuals ( 5 females, 2 males) remaining by the same person 20 years later.These people who still did not have any cognitive complaints that could affect their daily life activities continued their sociocultural relations and habits independently and were able to adapt to new information without much difficulty. During these 20 years, they did not experience cerebrovascular disease, head trauma, an infection that could affect any central nervous system, malignancy requiring immunosuppressive therapy, or severe chronic metabolicdegenerative disease. Besides, widespread white matter involvement and no parenchymal pathology were observed in cranial magnetic resonance imaging taken in the last six months.

Verbal memory processes test and Wechsler memory scale for visual memory assessment, digit span test for attention function, and verbal fluency, similarities (conceptualization), Stroop, and trail-making tests for evaluation of frontal lobe functions, Benton's line direction determination test for visuospatial organization and mini-mental state examination (MMSE) have been applied to individuals [25-34].

Cognitive tests were compared which were performed twenty years ago and now. IBM SPSS Statistics 25 program was used for data analysis. Paired Sample Test was used as well as determinative statistics such as mean and standard deviation, and $\mathrm{p}<0.05$ was considered statistically significant.

Ethics committee approval received from Haydarpaşa Numune Training and Research Hospital with decision number E-62977267-000-1150 in 25.01.2021.

\section{Results}

The average age of seven individuals included in the study was $75.71 \pm 4.89$ ( 5 female -2 male). When the test results are compared with the same cognitive tests performed twenty years ago, there was no significant difference in MMSE (mean: $28.29 \pm 0.76 / 26.71 \pm 2.36$ ) (p: 0.111 ). The most significant difference in the verbal memory processes test was in immediate memory results. A significant reduction of $45 \%$ was found in short-term memory scores (p:0,000*). On the other hand, impairment in recall-long-term memory (24\%) was less (P:0,029*). Twenty years ago, all fifteen words were recorded at an average of 7.71 repetitions, while now, during the test, all individuals had to do ten repetitions until the end of the test so that all words could be remembered. However, all fifteen words could be recorded without loss of words in total recall. In other words, all words could be recorded in memory (figure-1). 


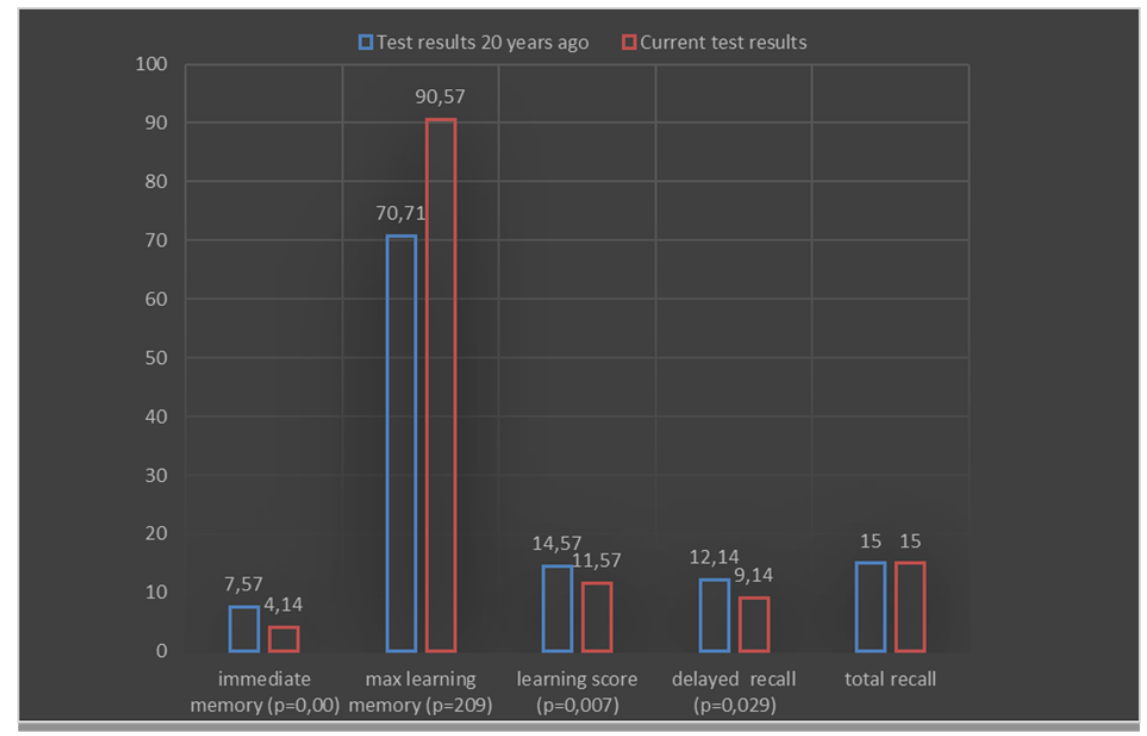

Figure 1 Verbal memory processes test results

In the WMS-R test in which visual memory is evaluated; it was observed that there was a $32 \%$ decrease in long-term visual recording memory ( $\mathrm{p}: 0,100)$ while the effect on instant visual recording memory was less (19\%) (p:0,274 ) (figure-2).

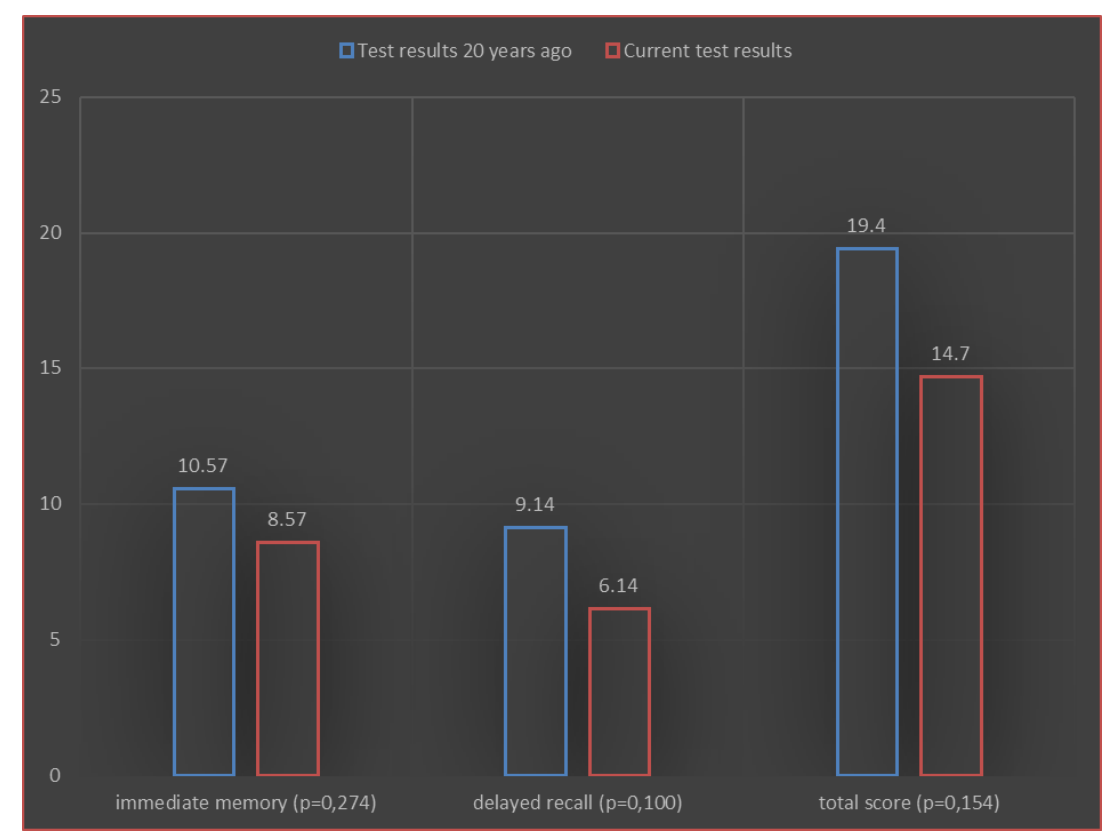

Figure 2 Visual memory scale test results

Attention was evaluated in series of numbers; in the first part, there was no change in the forward number range, while the effect on the more complex backward number range was greater, but there was a $10 \%$ decrease in this, which was not statistically significant ( $\mathrm{p}: 0,356)$. Considering the time difference between the two parts, it was observed that there was a moderate $(18 \%)$ prolongation in the current tests $(\mathrm{p}: 0,673)$.

Verbal fluency, Stroop, similarities (conceptualization), and trail-making tests were performed to evaluate frontal lobe functions (Table 1).

In the verbal fluency test made in three parts; in the first section where animal names were counted, there was a modest $(18 \%)$ decrease compared to tests twenty years ago $(\mathrm{p}: 0,160)$. When directed to a certain category (word count starting with the letter $\mathrm{K}$ ), it was observed that the improvement increased a little more (27\%) (p:0,040*). When the difficulty 
of the test increased even more and the function of category changing was added, the decrease in performance was found to be significantly lower at a rate of $33 \%$ (p: 0,019*).

Table 1 Tests evaluating the frontal lobe

\begin{tabular}{|l|l|l|l|l|l|}
\hline & & $\begin{array}{l}\text { Test results 20 } \\
\text { years ago }\end{array}$ & $\begin{array}{l}\text { Current test } \\
\text { results }\end{array}$ & $\begin{array}{l}\text { Percentage } \\
\text { change }\end{array}$ \\
\hline \multirow{2}{*}{ Verbal Fluency } & Animal name & $26,29 \pm 6,47$ & $21,43 \pm 7.04$ & 0,160 & $-\% 18$ \\
\cline { 2 - 6 } & Character K & $16,43 \pm 4,16$ & $11,86 \pm 4,02$ & $0,040 *$ & $-\% 27$ \\
\cline { 2 - 6 } & $\begin{array}{l}\text { Fruit-human } \\
\text { name }\end{array}$ & $9,43 \pm 2,88$ & $6,26 \pm 3,04$ & $0,019 *$ & $-\% 37$ \\
\hline \multirow{2}{*}{ Similarities } & & $20,43 \pm 1,81$ & $15,86 \pm 1,46$ & $0,002 *$ & $-\% 22$ \\
\hline \multirow{2}{*}{ Trail Making } & & $82,43 \pm 20,40$ & $207,43 \pm 57,09$ & $0,002 *$ & $+\% 151$ \\
\hline \multirow{2}{*}{ Stroop } & Reading & $26,29 \pm 5,22$ & $34,29 \pm 7,45$ & $0,002 *$ & $+\% 30$ \\
\cline { 2 - 6 } & Color reading & $87,28 \pm 28,74$ & $125,57 \pm 50,62$ & 0,057 & $+\% 44$ \\
\cline { 2 - 6 } & Time difference & $61,71 \pm 26,94$ & $91,29 \pm 52,58$ & 0,117 & $+\% 96$ \\
\hline
\end{tabular}

The change in the similarities test was also moderate, in which the difference between the two words given increasingly the degree of semantic difficulty in which abstraction was evaluated. There was also a $22 \%$ decrease in the achievement drive in this test $(\mathrm{p}: 0,002 *)$.

One person could not complete the test properly in the trial-making test which is more complex and difficult than other tests. In this test, complex attention, motor skills, planning and category changing abilities were evaluated. Test completion times were significantly longer when compared to twenty years ago (p:0,002).

In the Stroop test, which evaluated executive functions and was applied in two parts, there was $30 \%$ impairment in the straight reading section (p:0,002*). Although it was not statistically significant (44\%-p: 0.057), the decrease in performance in the colour reading section, where it was expected to continue the ability to change categories under a disincentive factor, was higher. Compared to the tests 20 years ago, the time difference between the two sections was $48 \%$ longer, although it was not statistically significant (p.0,117).

The decrease in Benton's line direction determination test was quite low (13\%) (p: 0.061) (Figure-3).

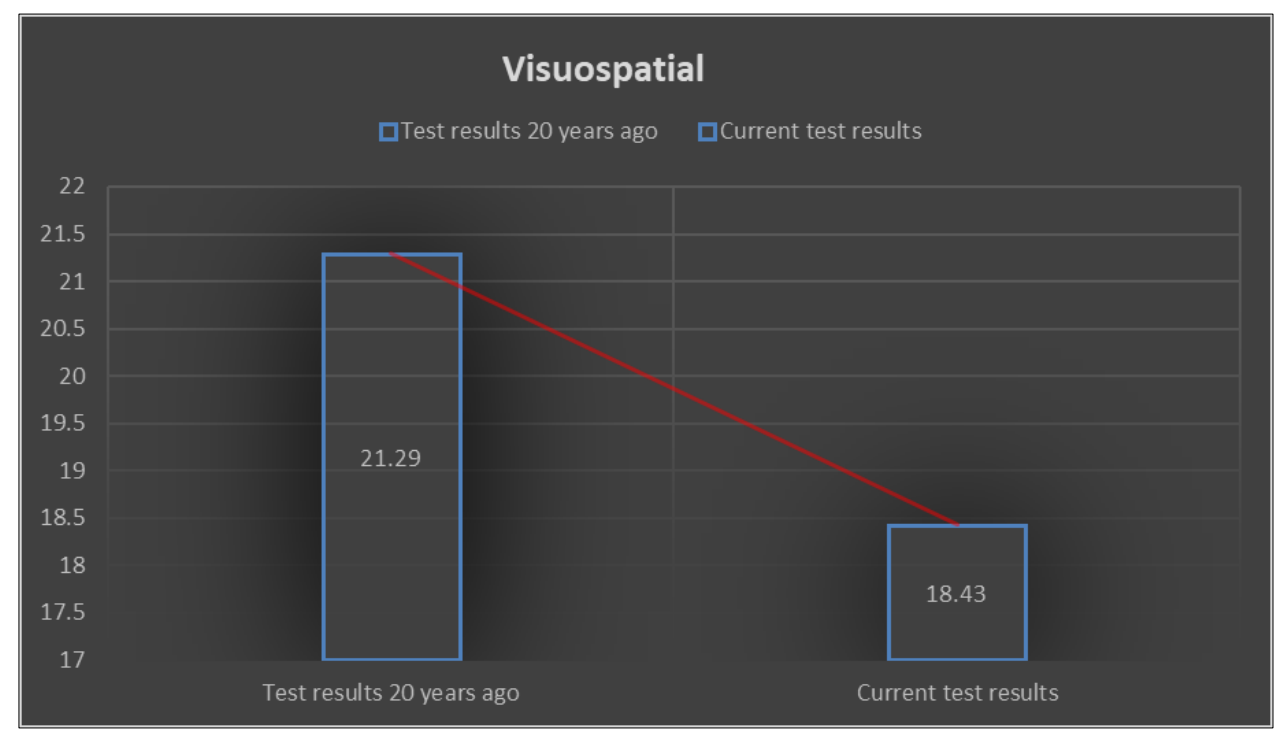

Figure 3 Benton's line direction determination test results 


\section{Discussion}

The negative effects of ageing on cognitive abilities are widely recognized and have been extensively studied in recent years $[12,18,24,26]$. As a result of the natural ageing process, age-related cognitive decline can also be observed in healthy individuals. The purpose of successful ageing is to maintain intact cognitive functioning until death [1]. It is important to understand how cognitive abilities change in healthy older adults, and this is the purpose of this study. Research in recent years on normal cognitive ageing has shown in detail how cognitive processes change with increasing age [1]. There is considerable variability in age-related cognitive changes from person to person, some of which can be attributed to genetic differences [3]. Normal cognitive ageing is not dementia and does not cause neuronal loss [1]. Many emotional functions are relatively preserved in ageing despite declines in various cognitive and physical functions. The ageing brain continues to adapt to stimuli, and some cognitive functions increase with age may compensate for functions that may decline although certain cognitive functions decline [5,18,35].

As people age, some neuroanatomical and neurophysiological modifications occur in the brain and there is ample evidence that those alterations in brain structure and function are intimately tied to alterations in cognitive function. Those cognitive alterations are visible for basic cognitive functions like attention (i.e., dividing and switching tasks), working memory, long-term memory and also for higher-level cognitive functions like language, decision-making and executive functioning. Other authors showed that, based on large sample sizes, while reasoning, visuospatial skills, memory and speed decline since 50 years old, vocabulary knowledge still increases until about 65 years old before decreasing. Thus, all forms of knowledge may not be sensitive in the same manner to age-related effects [36,37]. While some cognitive functions deteriorate with normal ageing, some are preserved [such as verbal skills, creativity] and some may be slightly affected. Due to the slowdown in processing speed during the normal ageing process, certain cognitive abilities such as memory, language, visuospatial function and executive function decrease [3]. There may be decreases in visual and verbal memory, abstraction, naming, verbal fluency and recall with slowing processing speed [5].Some cognitive abilities, such as vocabulary, are resistant to brain ageing and may even increase with age. Although language skills such as Word finding, naming and verbal fluency detoriorate with ageing, language skills such as producing meaningful word / sentences, verbal expression and grammer are preserved [1,3]. Episodic memory and working memory are most affected by ageing. In every decade, a 10\% reduction was expected in short term memory. However, large-scale population based studies document well-preserved memory functioning in some elderly individuals. The effective reserve concept argues that individual diffeences in brain characteristics or in the way people handle tasks allow some individuals to cope better and display preserved memory performance than others [38].

In our study; during the verbal memory processes test, all fifteen words were remembered among the multiple-choice clues at the end of the test, and they were all recorded with no loss of words although there was a $45 \%$ decrease in immediate memory compared to the values of twenty years ago, and ten repetitions were needed for all words to be remembered. Impairment in spontaneous recall (long-term memory) also remains 24\%. Visual memory impairment was less in immediate memory (19\%), but slightly more (32\%) in long-term memory.

Other abilities such as conceptual reasoning, memory, and processing speed gradually decrease over time. Studies have shown that concept formation, abstraction and mental flexibility decrease with age, especially after the age of 70 , because older adults tend to think more concretely than younger adults [3].

As a result of changes in perceptual systems or processing speed with normal ageing, cognitive abilities such as attention also deteriorate in addition to memory. Simple functions related to attention do not deteriorate significantly with ageing. However, divided attention function, which is more complex to deal with more than one aspect of a job, deteriorates with age. Cognitive ageing is not a disease or disorder, but a normal developmental process that occurs during adulthood. Older adults tend to perform better than younger adults in tasks where they can use the fund of knowledge they have experienced throughout their lives, such as some judgment and problem-solving tests [9,39]. In other words, the increase in a fund of knowledge leads to improvement in cognitive areas such as reasoning and decision-making in some general subjects. Many years of research in cognitive ageing psychology have identified agerelated patterns of changes in cognitive processes in which older adults performed worse on attention, working memory, and episodic memory tests than younger adults, and performed better on general knowledge tests [1].

In our study, there was a relatively moderate performance decrease at the rate of $22 \%$ in the similarity test, in which abstraction, reasoning and perception were evaluated. In the digit span test conducted to evaluate the attention function, there was no change in the repetition of straight numbers compared to twenty years ago, individuals were seen to have the same performance. With the process getting harder, there was only a $10 \%$ decrease in reverse number sequences and an $18 \%$ decrease in the completion time of the whole test. Our study showed that the affection in reasoning-abstraction abilities is moderate and relatively preserved, depending on attention and knowledge in 
individuals with normal ageing. Deterioration may occur with age in the visuospatial three-dimensional configuration which requires cognitive flexibility in spatial concentration [11]. We found a mild [13\%] impairment in the line direction determination test in our study.

The executive functions are also affected due to the changes in the dorsolateral prefrontal cortex in cognitive ageing, especially the decrease in the reactivity of processing speed and working memory [40]. It is suggested that the cognitive changes that occur due to normal ageing are mostly in executive functions. Executive functions are the skills that require cognitive flexibility such as planning, organizing, solving problems, detecting errors and making decisions. Like other cognitive abilities, decision making is affected by an age-related slowdown in information processing speed. However, cognitive capacity can be maintained and adequately expressed if the person is provided with sufficient time and clear explanations [40]. While the structural changes due to ageing are most prominent in the prefrontal cortex, medial temporal lobes are affected in 10-20\% of normal individuals. This finding supports the hypothesis that vocabulary is preserved in normal ageing. Elderly people continue to learn new words and concepts although they learn slowly [2,3].

In our study, for the tests evaluating executive functions and frontal lobe functions, performance decrease was not too much (18\%) than twenty years ago in the department where only animal names were counted based on vocabulary in verbal fluency. However, the decrease in the sections where the functions of a certain category (letter K) or category change [consecutive fruit-human name pairs] were evaluated was slightly more (27\%-33\%) by following the literature. The most striking affection is especially in the Stroop test, in which the effect of the slowdown in processing speed was observed significantly, and in the trail-making test due to the slowing of motor skills.

Adults may experience declines in cognition, affecting functional abilities and independence with increasing age [35]. The purpose of successful ageing is to maintain all cognitive functioning until death. Normal cognitive ageing is not dementia and does not cause neuronal loss, instead, there are changes in brain functioning [2,35]. We continue to learn with age, our knowledge does not decrease. However, there is a decrease in the ability to process information and recallremember it.

\section{Conclusion}

Cognitive functions based on attention, vocabulary and knowledge are substantially preserved with mild improvement in normal ageing. The affection in immediate memory is more specifically although long-term memory recall is relatively less affected. The most important improvement is on executive functions due to the decrease in motor and cognitive processing speed in cases where complex information needs to be processed.

\section{Compliance with ethical standards}

\section{Disclosure of conflict of interest}

No conflict of interest.

\section{Statement of informed consent}

Informed consent was obtained from all individual participants included in the study.

\section{References}

[1] Dumas JA. What is normal cognitive aging? Evidence from task-based functional neuroimaging. Curr Behav Neurosci reports. 2015; 2 [4]: 256-61.

[2] Salthouse TA. What and when of cognitive aging. Curr Dir Psychol Sci. 2004; 13 [4]: 140-4.

[3] Harada CN, Love MCN, Triebel KL. Normal cognitive aging. Clin Geriatr Med. 2013; 29[4]: 737-52.

[4] Casaletto KB, Elahi FM, Staffaroni AM, Walters S, Contreras WR, Wolf A, et al. Cognitive aging is not created equally: Differentiating unique cognitive phenotypes in "normal” adults. Neurobiol Aging. 2019; 77: 13-9.

[5] Fillit HM, Butler RN, O'Connell AW, Albert MS, Birren JE, Cotman CW, et al. Achieving and maintaining cognitive vitality with aging. In: Mayo Clinic Proceedings. Elsevier. 2002; 681-96.

[6] Keskin AO, Uncu G, Tanburoğlu A, Adapınar DÖ. Aging and senility related neurologic diseases. Osmangazi J Med. 2016; 38[1]: 75-82. 
[7] Açikgöz M. Assessment of Relation Between Subjectıve Memory Complaints and Objective Cognitive Performance of Elderly Over 55 Years Old Age. Nöro Psikiyatr Arşivi. 2014; 51[1]: 57.

[8] Nilsson L. Memory function in normal aging. Acta Neurol Scand. 2003; 107: 7-13.

[9] Dumas JA. Strategies for preventing cognitive decline in healthy older adults. Can J Psychiatry. 2017; 62 [11]: 75460.

[10] Luo L, Craik FIM. Aging and memory: A cognitive approach. Can J Psychiatry. 2008; 53[6]: 346-53.

[11] Drag LL, Bieliauskas LA. Contemporary review 2009: cognitive aging. J Geriatr Psychiatry Neurol. 2010; 23[2]: 75-93.

[12] Seidler RD, Bernard JA, Burutolu TB, Fling BW, Gordon MT, Gwin JT, et al. Motor control and aging: links to agerelated brain structural, functional, and biochemical effects. Neurosci Biobehav Rev. 2010; 34[5]: 721-33.

[13] Bergfield KL, Hanson KD, Chen K, Teipel SJ, Hampel H, Rapoport SI, et al. Age-related networks of regional covariance in MRI gray matter: reproducible multivariate patterns in healthy aging. Neuroimage. 2010; 49[2]: $1750-9$.

[14] Juraska JM, Lowry NC. Neuroanatomical changes associated with cognitive aging. Behav Neurobiol Aging. 2011; 137-62.

[15] Salthouse TA. Selective review of cognitive aging. J Int Neuropsychol Soc JINS. 2010; 16[5]: 754.

[16] Li X, Wang Y, Wang W, Huang W, Chen K, Xu K, et al. Age-related decline in the topological efficiency of the brain structural connectome and cognitive aging. Cereb Cortex. 2020; 30[8]: 4651-61.

[17] McDonough IM, Cervantes SN, Gray SJ, Gallo DA. Memory's aging echo: Age-related decline in neural reactivation of perceptual details during recollection. Neuroimage. 2014; 98: 346-58.

[18] St Jacques PL, Bessette-Symons B, Cabeza R. Functional neuroimaging studies of aging and emotion: Frontoamygdalar differences during emotional perception and episodic memory. J Int Neuropsychol Soc JINS. 2009; 15[6]: 819.

[19] Leal SL, Yassa MA. Neurocognitive aging and the hippocampus across species. Trends Neurosci. 2015; 38[12]: 800-12.

[20] Shankar SK. Biology of aging brain. Indian J Pathol Microbiol. 2010; 53[4]: 595.

[21] Polat F, Kumral E. Normal and pathologic brain aging.

[22] Bouazzaoui B, Follenfant A, Ric F, Fay S, Croizet J-C, Atzeni T, et al. Ageing-related stereotypes in memory: When the beliefs come true. Memory. 2016; 24[5]: 659-68.

[23] Ballesteros S, Mayas J, Reales JM. Cognitive function in normal aging and in older adults with mild cognitive impairment. 2014.

[24] Yu J, Collinson SL, Liew TM, Ng T-P, Mahendran R, Kua E-H, et al. Super-cognition in aging: cognitive profiles and associated lifestyle factors. Appl Neuropsychol Adult. 2019.

[25] Folstein MF, Folstein SE, McHugh PR. "Mini-mental state": a practical method for grading the cognitive state of patients for the clinician. J Psychiatr Res. 1975; 12[3]: 189-98.

[26] Öktem Ö. Sözel Bellek Süreçleri Testi, Bir ön çalışma. Nöropsikiyatri Arşivi. 1992; 29.

[27] Loring DW. The Wechsler memory scale-revised, or the Wechsler memory scale-revisited? Clin Neuropsychol. 1989; 3[1]: 59-69.

[28] Elwood RW. The Wechsler Memory Scale-Revised: psychometric characteristics and clinical application. Neuropsychol Rev. 1991; 2[2]: 179-201.

[29] Öktem Ö. Nöropsikolojik testler ve nöropsikolojik değerlendirme. Türk Psikol Derg. 1994; 9[33]: 33-44.

[30] Dubois B, Slachevsky A, Litvan I, Pillon B. The FAB: a frontal assessment battery at bedside. Neurology. 2000; 55[11]: 1621-6.

[31] Stroop JR. Studies of interference in serial verbal reactions. J Exp Psychol. 1935; 18[6]: 643.

[32] MacLeod CM, MacDonald PA. Interdimensional interference in the Stroop effect: Uncovering the cognitive and neural anatomy of attention. Trends Cogn Sci. 2000; 4[10]: 383-91. 
[33] Cangoz B, Karakoc E, Selekler K. Trail Making Test: normative data for Turkish elderly population by age, sex and education. J Neurol Sci. 2009; 283[1-2]: 73-8.

[34] Benton AL, Varney NR, Hamsher K deS. Visuospatial judgment: A clinical test. Arch Neurol. 1978; 35[6]: $364-7$.

[35] Pressman PS, Noniyeva Y, Bott N, Dutt S, Sturm V, Miller BL, et al. Comparing volume loss in neuroanatomical regions of emotion versus regions of cognition in healthy aging. PLoS One. 2016; 11[8]: e0158187.

[36] Anderson ND, Craik FIM. 50 years of cognitive aging theory. Journals Gerontol Ser B. 2017; 72[1]: 1-6.

[37] Calso C, Besnard J, Allain P. Frontal lobe functions in normal aging: metacognition, autonomy, and quality of life. Exp Aging Res. 2019; 45[1]: 10-27.

[38] Nyberg L, Lövdén M, Riklund K, Lindenberger U, Bäckman L. Memory aging and brain maintenance. Trends Cogn Sci. 2012; 16[5]: 292-305.

[39] Salthouse TA. Trajectories of normal cognitive aging. Psychol Aging. 2019; 34[1]: 17.

[40] Tannou T, Koeberlé S, Aubry R, Haffen E. How does decisional capacity evolve with normal cognitive aging: systematic review of the literature. Eur Geriatr Med. 2020; 11[1]: 117-29. 